\title{
Metagenome-scale analysis yields insights into the structure and function of microbial communities in a copper bioleaching heap
}

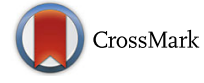

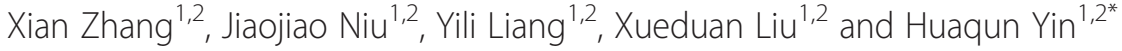

\begin{abstract}
Background: Metagenomics allows us to acquire the potential resources from both cultivatable and uncultivable microorganisms in the environment. Here, shotgun metagenome sequencing was used to investigate microbial communities from the surface layer of low grade copper tailings that were industrially bioleached at the Dexing Copper Mine, China. A bioinformatics analysis was further performed to elucidate structural and functional properties of the microbial communities in a copper bioleaching heap.
\end{abstract}

Results: Taxonomic analysis revealed unexpectedly high microbial biodiversity of this extremely acidic environment, as most sequences were phylogenetically assigned to Proteobacteria, while Euryarchaeota-related sequences occupied little proportion in this system, assuming that Archaea probably played little role in the bioleaching systems. At the genus level, the microbial community in mineral surface-layer was dominated by the sulfur- and iron-oxidizing acidophiles such as Acidithiobacillus-like populations, most of which were A. ferrivorans-like and A. ferrooxidans-like groups. In addition, Caudovirales were the dominant viral type observed in this extremely environment. Functional analysis illustrated that the principal participants related to the key metabolic pathways (carbon fixation, nitrogen metabolism, Fe(II) oxidation and sulfur metabolism) were mainly identified to be Acidithiobacillus-like, Thiobacillus-like and Leptospirillum-like microorganisms, indicating their vital roles. Also, microbial community harbored certain adaptive mechanisms (heavy metal resistance, low pH adaption, organic solvents tolerance and detoxification of hydroxyl radicals) as they performed their functions in the bioleaching system.

Conclusion: Our study provides several valuable datasets for understanding the microbial community composition and function in the surface-layer of copper bioleaching heap.

Keywords: Metagenomics, Taxonomic analysis, Functional analysis

\section{Background}

The microbial communities in mine tailings have attracted considerable interest, and there are many relevant microbiological researches of mine tailings, most of which have been performed in the last decade [1,2]. Previous cultivation-dependent studies of mine tailings in several countries have revealed a numerical dominance of Bacteria over Archaea. Particularly abundant microorganisms were acidophilic iron- and/or sulfuroxidizing Acidithiobacillus and Leptospirillum [3-6]. In

\footnotetext{
* Correspondence: yinhuaqun@gmail.com

${ }^{1}$ School of Minerals Processing and Bioengineering, Central South University, Changsha, China

${ }^{2}$ Key Laboratory of Biometallurgy of Ministry of Education, Central South University, Changsha, China
}

addition, metagenomic analysis revealed that the most abundant microorganisms in a low-temperature acid mine drainage stream were most similar to the psychrotolerant acidophile, Acidithiobacillus ferrivorans [7]. However, studies on the extremely acidic lead/zinc mine tailings revealed that acidophilic archaea, mostly the ferrous-iron-oxidizing Ferroplasma acidiphilum, were numerically significant, indicating their importance in extremely acidic environments [8]. Furthermore, previous studies indicated that $\mathrm{pH}$ was the primary factor in shaping the microbial community structure at different acidification stages of mine tailings [9].

Heap bioleaching has been successfully used to extract basic metals from sulfide minerals [10]. Copper bioleaching for commercial applicationthat was designed to exploit 
microbial activities was pioneered in 1980 [10]. Copper mine tailings with low-grade was used for bioleaching heaps in the field of industrial applications, representing a type of acidic environments of anthropogenic origin [11]. In the process of industrial bioleaching operations, insoluble metal sulfides were converted into water-soluble metal sulfates, resulting in the considerably high concentrations of soluble iron and meanwhile low $\mathrm{pH}$. As reviewed by Bonnefoy and Holmes (2012), ferrous iron [Fe(II)] was oxidized rapidly to generate ferric iron $[\mathrm{Fe}(\mathrm{III})]$ under oxygen-saturated conditions with neutral $\mathrm{pH}$, whilst in acidic environments, $\mathrm{Fe}(\mathrm{II})$ was stable even under the condition of existence of atmospheric oxygen $\left(\mathrm{O}_{2}\right)$. Thus, $\mathrm{Fe}(\mathrm{II})$ could be utilized by microbial communities as the electron donor. Nonetheless, the highest levels of soluble iron could challenge microorganisms living in the extremely acidic environments. Hydroxyl radicals produced by the reaction of free ferrous iron with oxygen (Fenton reaction) would damage biological macromolecules and even cause cell death [12]. The extremely acidic environments provide a particular opportunity, and meanwhile, a potential challenge for life. In recent decades, issues associated with life in the oligotrophic, extremely acidic environments have been discussed in a number of reviews and papers, including the occurrence and composition of microbial communities [13-15], their strategies to tolerate the metal and low $\mathrm{pH}[16,17]$, as well as their metabolisms and functions $[18,19]$.

The microbial ecology of full-scale heap or dump bioleaching of copper ore has been poorly understood [20]. As stated by Brierley (2001), the understanding of microbiological components of bioheaps facilitated commercial bioheap applications. Although 16S rRNA gene analysis was targeted as a useful method in many studies of extreme environments, the development of sequencing technology, metagenomics methods and bioinformatics tools have provided a valuable platform for environmental gene pool identification and potential functional prediction of biogeochemical relevance in the microbial populations [21]. Metagenomics, or the culture-independent genomic analytical method of microorganisms, was a powerful approach to capture the entire spectrum of microbial communities including both cultivatable and uncultivable microorganisms, the latter of which could not be cultured by standard techniques but comprised the majority of biological diversity [22-25]. Metagenomic research associated with ecological roles of uncultured and rare microorganisms showed their importance in acid mine drainage (AMD) communities [26]. A combination of shotgun metagenome sequencing and computational approaches for genome assembly has advanced to metagenomics, providing glimpses into the uncultured microbial world [27].

In this study, we collected samples from the surfacelayer mine tailings of bioleaching heap located in the
Dexing Copper Mine, Jiangxi Province, China. By investigating the taxonomic classification and functional genes involved in several key metabolic processes, based on metagenome analyses, we sought to characterize the microbial community composition in the bioleaching dumps heaped up by mine tailings and the functional coding potential of microorganisms related to key metabolic pathways within the extremely acidic environmental conditions.

\section{Results and discussion}

\section{Sequencing, de novo assembly, gene prediction and} functional annotation

Metagenomic DNA was subjected to Illumina MiSeq sequencing, and approximately 3.4 million short DNA sequences were then used for bioinformatics analysis. After quality control using NGS QC Toolkit, 2,941,297 (87.80 \%) reads with high-quality were obtained (Additional file 1). Subsequently, all high-quality reads aforementioned were assembled, and a self-writing script was used to filter the assembled sequences under $300 \mathrm{bp}$, resulting in a total of 301,907,459 bases, with an N50 of 641 bp (481,688 contigs range from 301 bp to $49,868 \mathrm{bp}$, and the mean length was $626 \mathrm{bp}$ ). For gene prediction, 660,572 coding sequences (CDS) were identified using the program MetaGeneAnnotator.

All putative protein coding sequences were searched against the databases including NCBI-nr, the extended COG [28] and KEGG, and we obtained a total of 535,887 (81.12 \%), 517,948 (78.41 \%) and 494,721 (74.89\%) significant BLAST hits respectively. Moreover, 497,601 (75.33 \%) and 261,595 (39.60 \%) sequences were assigned to the COG categories and KEGG Orthology respectively (Additional file 1). Among the 25 COG categories, metagenome sequences were assigned to 23 of them (Fig. 1). A large proportion of sequences were assigned to COG category [S] (function unknown) (80,561 CDSs; $16.19 \%$ ) and COG category [R] (General function prediction only) $(39,507$ CDSs; $7.94 \%)$, indicating large pools of potential unknown functional genes in copper bioleaching operations. Furthermore, the large amount of genes associated with basal metabolisms such as amino acid transport and metabolism (COG category [E]) and energy metabolism (COG category $[\mathrm{C}]$ ) indicated the ubiquitous substance and energy metabolism in the extremely environments, maintaining the basic microbial activities.

\section{Taxonomic assignment of metagenome datasets}

To reveal metagenome sequence classification of microbial communities in tailings sample, taxonomic analyses at the genus level were performed. Taxonomic assignment using the program MEGAN revealed unexpectedly abundant microbial biodiversity (over 100 


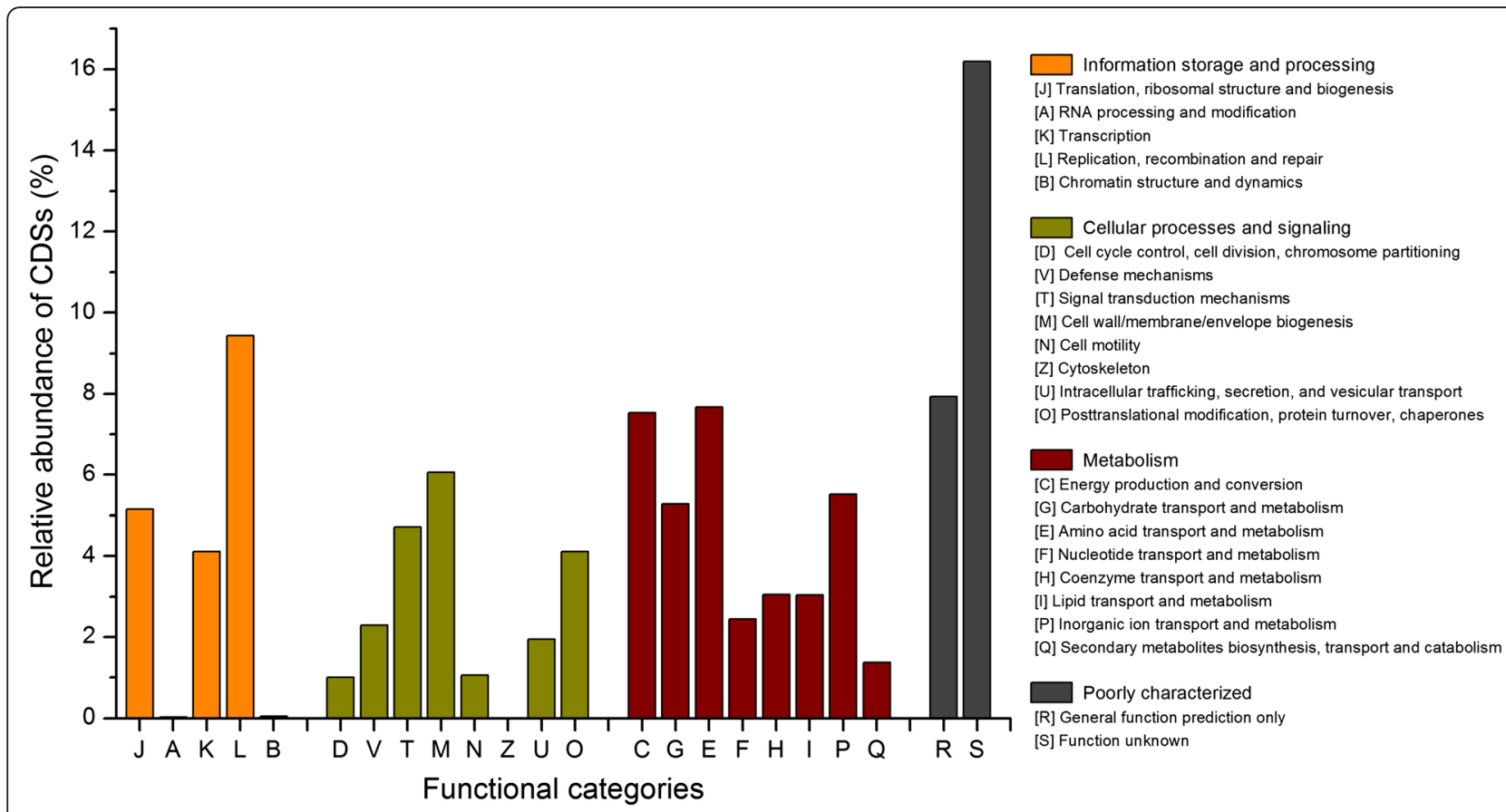

Fig. 1 The COGs categories of metagenome data from mine tailings

genera) of this extreme environments (surface-layer of copper mine tailings), to some extent, which hindered the sequence assembly due to the low sequencing depth. Copper mine tailings in this study harbored diverse microbial populations possibly because of various niches related to gradients of physico-chemical conditions, which was discussed previously in AMD environments [2, 29-31]. MEGAN analysis showed that the microbial community in mineral surface-layer was dominated by the sulfur- and iron- oxidizing acidophiles Acidithiobacillus-related and Leptospirillum-related groups (Fig. 2). In these Acidithiobacillus-related sequences, most of them were assigned to Acidithiobacillus ferrivorans, followed closely by A. ferrooxidans. In

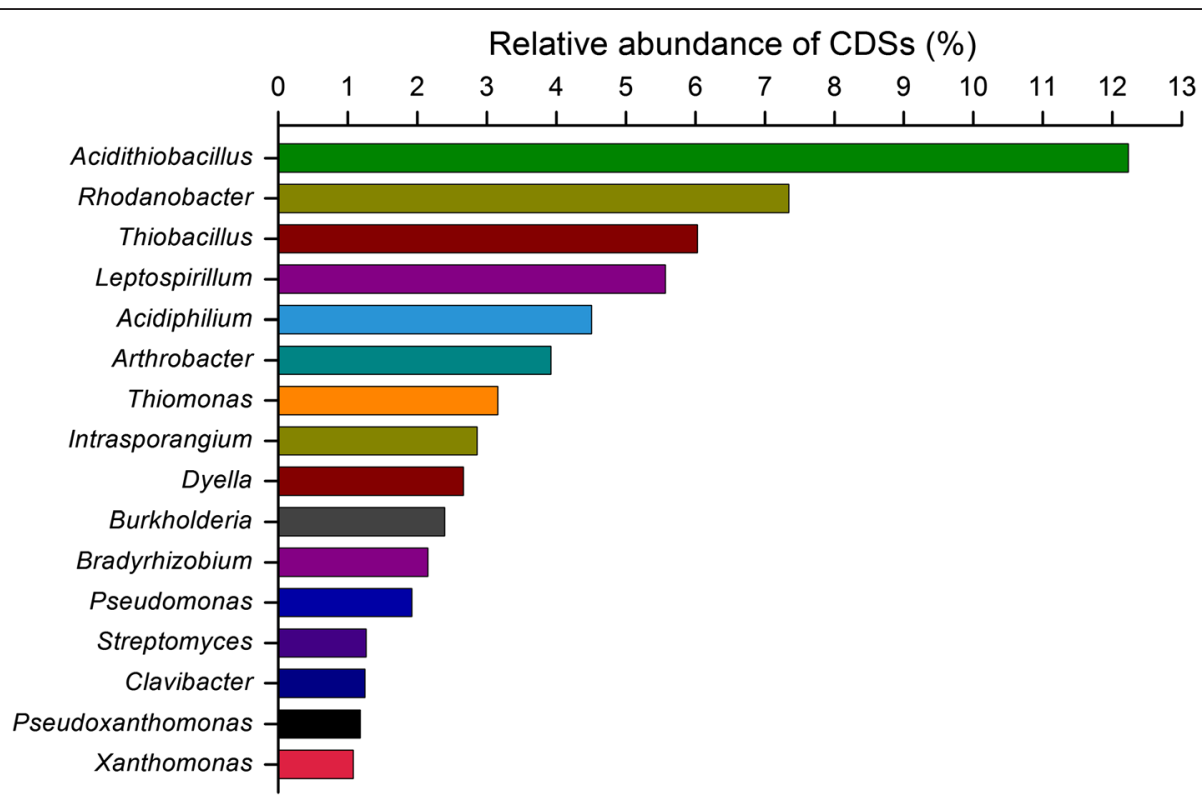

Fig. 2 Taxonomic composition analysis at the genus level based on contigs sequences ( $\geq 300 \mathrm{bp}$ ) in the metagenome dataset. Only those genera with the specified percentage abundance ( $\geq 1 \%$ ) are shown 
the extremely acidic tailings, approximately $93.47 \%$ of the total Acidithiobacillus-related sequences were affiliated with $A$. ferrivorans and A. ferrooxidans (Additional file 2). As a major participant of iron- and sulfur-oxidizing acidophilic bacteria, A. ferrivorans has been widely found in metal mine-impacted environments [32]. Likewise, $A$. ferrooxidans, which utilized energy from the oxidation of sulfur- and iron-containing minerals, was a principal member in consortia of microorganisms associated with the bioleaching or biomining (industrial recovery of copper) [33]. The numerical dominance of Acidithiobacillus-related sequence indicated its importance in surface-layer of copper mine tailings during the industrial bioleaching operations. Moreover, Rhodanobacter (7.34 \%), Thiobacillus (6.03\%), Leptospirillum (5.57\%), and Acidiphilium (4.51\%) were also found in the surface-layer mine tailings. In addition, 82 CDSs were assigned to virus, most of which were affiliated with the dsDNA viruses with no RNA stage. Of these sequences, the majority of taxonomic hits $(74 \%)$ shared sequence identity with sequences in the order Caudovirales, based on the taxonomy of viral genomes provided by GenBank database (Additional file 3). This was consistent with the viruses previously described from the desert [34, 35] and other metaviromes from other environments such as marine environment [36].

Depend on the automated analysis pipeline implemented in the MG-RAST platform, the microbial populations at the phylum level were phylogenetically assigned to the Proteobacteria, Actinobacteria, Nitrospirae, Bacteroidetes, Gemmatimonadetes, Acidobacteria, Firmicutes, Deinococcus-Thermus, Euryarchaeota, and several other phyla mainly belonged to the domain Bacteria (Additional file 4). In more detail, Proteobacteria-related sequences with the most abundance were composed of the class Gammaproteobacteria, Betaproteobacteria, Alphaproteobacteria, Deltaproteobacteria, Epsilonproteobacteria and Zetaproteobacteriain an order from the highest to the lowest. Similarly, community diversity analysis based on a PCR-based cloning approach showed that the majority of sequenced clones were affiliate with the Gammaproteobacteria [37]. As the most abundant microbes were similar to Acidithiobacillus-like genus, it was proposed to belong to the new class Acidithiobacillia (a sister group of class Gammaproteobacteria) [38]. Thus, the most abundant sequences at the class level in this extreme environment could be assigned to the Acidithiobacillia-related microorganisms. The phylum Euryarchaeota occupied the largest proportion in domain Archaea. However, it was relatively low in the whole metagenome dataset, suggesting that the Archaea might play little role in the surface-layer of copper mine tailings.

\section{Key genes coding for enzymes associated with principal metabolisms}

The vital activities of chemolithotrophy-based microbial community present in the mine tailings mainly rely on metabolic capabilities to metabolize carbon, nitrogen, iron and sulfur. Thus, it is necessary to investigate the general metabolisms of microbial processes, aiming to understand the sub-cycling of those elements within a copper bioleaching heap.

\section{Autotrophic carbon fixation}

Cellular carbon acquired from inorganic carbon is essential for life, suggesting the transition of carbon from inorganic to organic world. Recent research revealed that six different pathways for carbon fixation existed in microorganisms, including Calvin-Benson-Bassham (CBB) cycle, reductive citric acid (rTCA) cycle, reductive acetylcoenzyme A (acetyl-CoA) pathway, 3-hydroxypropionate bicycle, 3-hydroxypropionate/4-hydroxybutyrate cycle (hydroxypropionate-hydroxybutyrate cycle) and dicarboxylate-4-hydroxybutyrate cycle (shortened to the dicarb oxylate-hydroxybutyrate cycle) [2,39]. Functional annotation against databases, i.e., NCBI-nr, the extended COG and KEGG, showed that approximately all genes encoding for $\mathrm{CBB}$ cycle and rTCA cycle were identified, whilst no gene involved in the other four pathways for carbon fixation was identified. This finding was largely consistent with previous studies [2].

In $\mathrm{CBB}$ cycle, there are a series of enzymatic reactions, one of which is involved in $\mathrm{CO}_{2}$ fixation catalyzed by ribulose-1,5-bisphosphate carboxylase/oxygenase (Rubisco) and the others are responsible for the regeneration of ribulose 1,5-bisphosphate (RuBP) [40]. In addition, the key enzymes for CBB cycle are Rubisco and phosphoribulokinase (or ribulose-5-phosphate kinase) [39, 41]. Rubisco exists in various forms in diverse organisms from all domains of life [42]. Our results indicated that genes coding for Rubisco were enriched in the metagenome, most of which were affiliated with Acidithiobacillus-like populations (Additional file 5). Indeed, genomic as well as proteomic evidence showed that Acidithiobacillus occupied an important position in carbon fixation in AMD [43]. In addition, the majority of phosphoribulokinase (PRK) genes were from Thiobacillus-like microorganisms, indicating its importance in the bioleaching system. However, no gene encoding the sedoheptulose-1,7-bisphosphatase (SBPase), which catalyzed sedoheptulose 1,7-bisphosphate to generate sedoheptulose 7-phosphate, was found in the metagenome (Additional file 5). The lack of such genes was likely due to the low sequencing depth. Besides, the candidate genes that probably presented in this metagenome dataset were not yet identified because 
of our limited knowledge of SBPase genes within the acidophiles.

Autotrophic $\mathrm{CO}_{2}$ fixation via rTCA cycle was considered to be an important pathway in microbial communities [44]. There were two key enzymes (2-oxoglutarate ferredoxin oxidoreductase and ATP citrate lyase) related to rTCA cycle [39]. In some species, the citrate cleavage pathway could be catalyzed by other two enzymes, citrylCoA synthetase (EC6.2.1.18) and citryl-CoA lyase (EC4.1.3.34), instead of ATP citrate lyase (ACL; EC2.3.3.8) [45]. These two enzymes, i.e., citryl-CoA synthetase and citryl-CoA lyase, however, were phylogenetically related to ACL [46-48]. Furthermore, research indicated that ACL was formed by a gene fusion of citryl-CoA lyase and citryl-CoA synthetase [48]. These findings could explain why no genes encoding ACL were identified in previous studies about lead/zinc mine tailings [49]. In Leptospirillum ferriphilum, a complete set of enzymes involved in rTCA cycle were found [40]. Based on the aforementioned research, our results in this study showed that gene homologs for many steps in the rTCA cycle were assigned to the $L$. ferriphilum-like populations (Additional file 5). As for citryl-CoA synthetase, however, the small subunit gene $(\operatorname{css} B)$ was not detected.

\section{Nitrogen metabolism}

As the main nitrogen sources, generally speaking, atmospheric nitrogen, nitrate, nitrite, ammonium and glutamine are used wildly by microbes in natural environments. Six subsystems related to the nitrogen metabolisms, including nitrogen fixation, dissimilatory nitrate reduction, assimilatory nitrate reduction, denitrification, nitrification and anammox, were discussed in previous papers [50,51]. In this metagenome, a large number of genes involved in nitrogen fixation including nifD, nifK, nifH, nifE, nif $N$ and nifX, which formed a nif operon encoding the Mo-Fe nitrogenase enzyme complex [52], were detected (Additional file 5). The relevant genes were largely found in Leptospirillum-like and had the highest similarity to those from L. ferrooxidans-like species, suggesting the vital position of Leptospirillum-like organisms in bioleaching systems with limited fixed nitrogen from external sources.

Nitrogen in the form of ammonium could be either directly assimilated into biomass or be oxidized by two key enzymes involved in the nitrification (ammonium monooxygenase and hydroxylamine oxidoreductase, which were encoded by $a m o C A B$ operon and hao gene, respectively) [52]. In this metagenome, however, the lacked hao gene suggested that ammonium could not be utilized via the nitrification. Mine tailings might contain elevated concentrations of nitrate caused by the nitrogen-based explosives. As for dissimilatory nitrate reduction and assimilatory nitrate reduction, most genes encoding metabolic enzymes were related to those from Thiobacillus-like, Acidithiobacillus-like and Leptospirillum-like populations (Fig. 3). Given that there was limited fixation nitrogen from atmosphere and relatively low content of available nitrogen in mine tailings, it was speculated that these microorganisms probably played an important role in maintaining the nitrogen sources of microbial communities via utilizing nitrate and nitrite.

Four enzymes are reported to be involved in denitrification i.e., nitrate reductase, nitrite reductase (NO-forming), nitric oxide reductase (cytochrome $\mathrm{c}$ ) and nitrous oxide reductase, which are encoded by nar operon, nirK, nor BC and $n o s Z$, respectively $[2,53]$. In this process, nitrate or nitrite ions could be used as the terminal electron acceptors under anoxic or low-oxygen conditions [52]. In addition, the incomplete ammonification in this system, which lacked the relevant genes encoding hydrazine oxidoreductase (Fig. 3), presumably indicated that organic nitrogen compounds in the environments could not be degraded by microbes in the bioleaching system.

\section{Ferrous iron oxidation}

Under acidic conditions, ferrous iron in a stable status (no matter whether atmospheric oxygen exists or not) could be utilized by microorganisms [11]. Widespread distribution of ferrous iron oxidation capabilities has been observed in acidophilic Bacteria and Archaea [54-56]. The ability to oxidize iron has been widely distributed in microbes from neutral $\mathrm{pH}$ environments. So far, details of ferrous iron oxidation and electron transport pathways were available for the Gram-negative bacterium A. ferrooxidans that was identified until recently to be affiliated with the class Acidithiobacillia [38]. Besides, iron oxidation pathways in other known acidophilic prokaryotes (e.g., $A$. ferrivorans, L. ferrooxidans and Thiobacillus prosperus, which was reclassified as Acidihalobacter prosperus recently [57]) have been also studied [11]. The results showed that there were no relevant genes for the pio operon (pioA, pioB, and pioC) or the fox operon (foxE, fox $Y$ and $f \circ x Z$ ) in the metagenome, which were reported to be involved in the phototrophic iron oxidation in Rhodopseudomonas palustris and Rhodobacter capsulatus respectively [2, 58]. The reasons why the genes for ferrous iron oxidation were absent in acidophilic microbes were probably (i) due to the low abundance of microorganisms responsible for iron oxidation, resulting in the difficulty in the retrieval of relevant genes from metagenomeand (ii) due to limited knowledge of iron oxidation in microorganisms $[2,11]$.

Genes for the redox proteins involved in $\mathrm{Fe}(\mathrm{II})$ oxidation in acidophiles (such as outer membrane cytochrome $c$ or Cyc2, blue copper proteins rusticyanin and $\mathrm{Cyc1}$, etc.) 


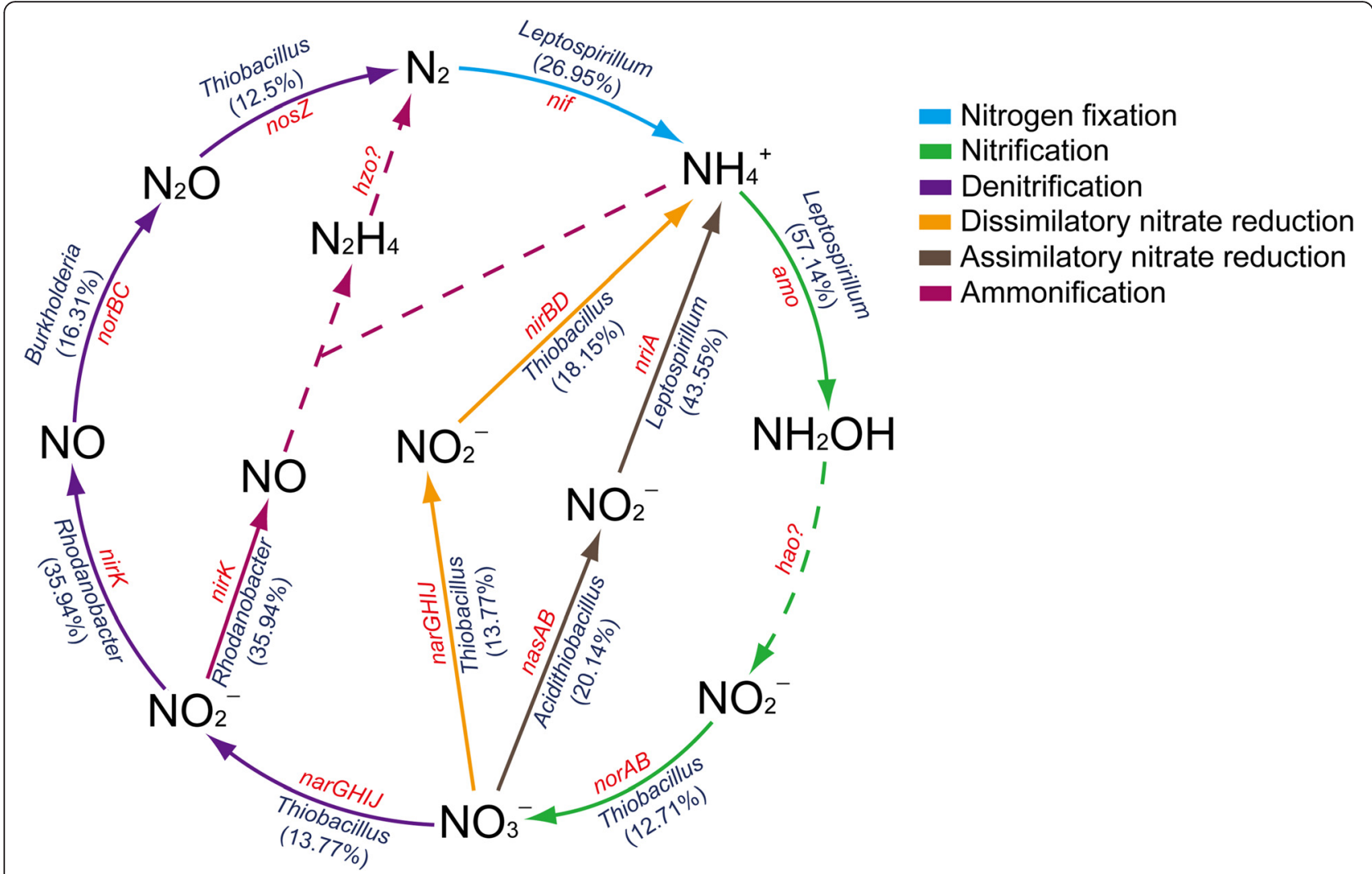

Fig. 3 Schematic diagram of nitrogen metabolism in the surface-layer mine tailings representing one important part of bioleaching system. On the basis of metagenomic data, solid lines indicate the presence of protein-coding genes associated with six major pathways, whereas dashed lines show that no gene was found in this metagenome. Different colored lines depict various metabolic pathways. Most main implicated taxa/groups governing the enzymatic reactions are displayed respectively, and the percentages of CDSs related to each group are also showed. Abbreviations: nif, nitrogenase (various subunits); amo: ammonia monooxygenase; hao: hydroxylamine dehydrogenase; nor $A B$ : Nitrate reductase (A and B represent alpha subunit and beta subunit, respectively); narGHIJ: nitrate reductase (dissimilartory); nirK: nitrite reductase (NO-forming); norBC: nitric oxide reductase; nosZ: nitrous oxide reductase; nasAB: nitrate reductase (assimilatory); nirA: ferredoxin-nitrite reductase; nirBD: nitrite reductase (NADH); hzo:

hydrazine oxidoreductase

$[2,11,58,59]$ were observed in this study (Fig. 4). The results indicated that rusticyanin genes (rus) existed in the metagenome, most of which were assigned to $A$. ferrooxidans-like species. Moreover, it also exhibited genes encoding iron oxidase in $A$. ferrivorans-like, though their number was not enriched. Thus, there were at least two different pathways for ferrous iron oxidation in the iron-oxidizing Acidithiobacillus spp.: (i) via rusticyanin (ii) or via high potential iron sulfur protein (HiPIP) encoded by the gene iro [11]. In acidophilic archaea, $\mathrm{Fe}(\mathrm{II})$ oxidation pathways were also proposed $[11,52,60]$, indicating that electrons from Fe(II) oxidation by unknown mechanisms were transferred via sulfocyanin to a $c b b_{3}$-type terminal oxidase (Fig. 4). In the tailings sample, only one sulfocyanin gene in the archaean genus Ferroplasma-like was identified (Additional file 5). Given the low relative abundance of Archaea in surface-layer of mine tailings, it was supposed that Archaea might play little role in the $\mathrm{Fe}(\mathrm{II})$ oxidation within the mine tailings.

\section{Sulfur metabolism}

Recently, there are a number of known enzymes that are related to the sulfur metabolism by microoorganisms [61-63]. Undoubtedly, the transformation of elemental sulfur with various oxidation states is more complicated than iron metabolism. In surface-layer of bioleaching heap, the genes encoding sulfur metabolic enzymes have not yet been elaborated. In this study, a total of 2479 CDSs were found to be linked to sulfur metabolism, mapping to 48 diverse genes (Additional file 5). Sulfur and reduced inorganic sulfur compounds (RISCs) might accumulate in areas where pyrite and other types of sulfide minerals were oxidized by $\mathrm{Fe}(\mathrm{III})$ ion [64]. Thus, the energetically favorable substrates could be utilized by acidophilic sulfur-oxidizing bacteria $[62,63]$. Microbial oxidation of sulfur or sulfur-compounds requires different enzymatic machineries, including sulfide quinone reductase (SQR), thiosulfate:quinone oxidoreductase (TQO) and tetrathionate hydrolase (TetH) (Fig. 4). 


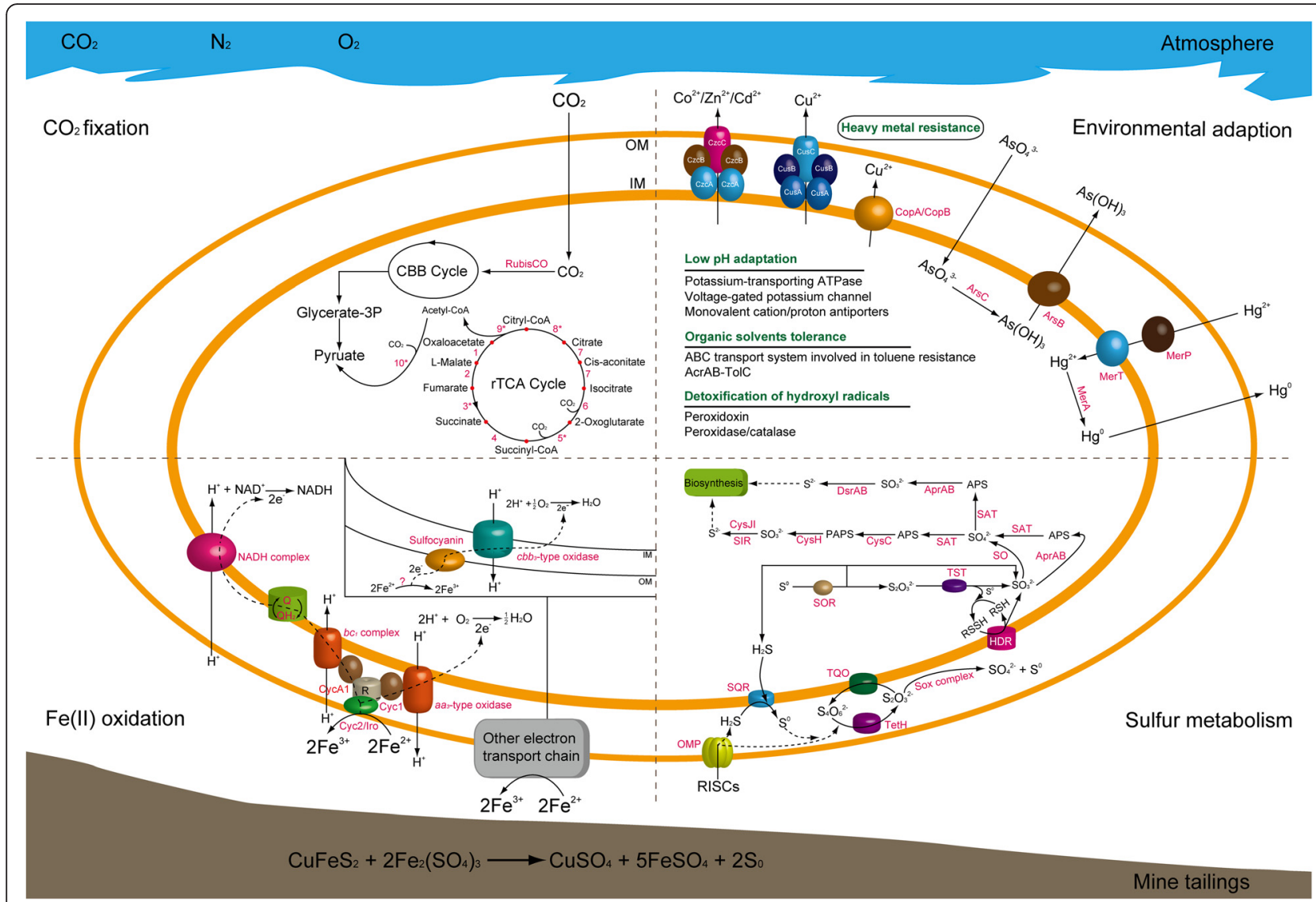

Fig. 4 Overview of the main known metabolic abilities (carbon fixation, ferrous iron oxidation and sulfur metabolism) of microbial community and environmental adaption in surface-layer mine tailings. This figure was adapted from the previous models $[26,40,52,61]$. All possible subsystems are depicted in the quarters of each image. In the $\mathrm{CO}_{2}$ fixation, enzymes associated with rTCA cycle are indicated by numbers: 1, malate dehydrogenase; 2, fumarate hydratase; 3, fumarate reductase; 4, succinyl-CoA synthetase; 5, 2-oxoglutarate ferredoxin oxidoreductase; 6, isocitrate dehydrogenase; 7 , aconitase hydratase 1; 8, citryl-CoA synthetase; 9, citryl-CoA lyase; 10, pyruvate ferredoxin oxidoreductase. The enzymes related to nitrogen metabolism, ferrous iron oxidation and sulfur metabolism as abbreviated forms are depicted. Abbreviations: R: Rusticyanin; SQR: sulfide quinone reductase; TQO: thiosulfate:quinone oxidoreductase; TetH: tetrathionate hydrolase; SOX: sulfur oxidizing protein; HDR: heterodisulfide reductase; SOR: sulfur oxygenase reductase; TST: thiosulfate sulfurtransferase; SO: sulfite oxidase; APR: adenylylsulfate reductase; SAT: sulfate adenylyltransferase; CysC: adenylylsulfate kinase; CysH: phosphoadenosine phosphosulfate reductase; CysJl: sulfite reductase (NADPH) flavoprotein; SIR: sulfite reductase (ferredoxin); DsrAB: sulfite reductase

As an intermediate forms during the RISC oxidation, sulfide was oxidized by SQR, the coding genes of which were enriched in the metagenome and were most closely identified as those of Acidithiobacillus-like populations, to generate elemental sulfur. In addition, the enriched genes encoding TQO, which catalyzed thiosulfate to produce tetrathionate, were also closely related to Acidithiobacillus-like genus. As the substrate of TetH, tetrathionate could be further hydrolyzed to thiosulfate, sulfate and elemental sulfur [61]. The genes encoding the truncated sulfur oxidation protein (Sox) system (SoxAX, SoxB and SoxYZ) were identified in Acidithiobacillus-like and Thiobacillus-like microorganisms. Although not enriched, Sox $C D$ genes were also detected in the metagenome and identified in Rhodanobacter-like and Thiomonas-like populations. Sulfur oxygenase reductase (SOR) reported previously in certain bacteria such as Acidithiobacillus thiooxidans and A. caldus [61, 65] and thermo-acidophilic archaea such as Acidianus tengchongensis and A. ambivalens [66, 67] were found in the metagenome. In addition, the genes encoding heterodisulfide reductase $(h d r A B C)$ and thiosulfate sulfurtransferase $(t s t)$, which participated in the catalysis of thiosulfate to sulfite, were abundant in Acidithiobacilluslike populations. Sulfite would be oxidized directly by sulfite oxidase to sulfate or be catalyzed by adenylylsulfate (APS) reductase and sulfate adenylyltransferase (SAT) to generate sulfate via the reversed dissimilatory sulfuate reduction. Remarkably, the $\operatorname{apr} A B$ genes encoding APS reductase were enriched in the metagenome, most of which were assigned to those of Thiobacilluslike populations. No such gene, however, was found in 
Acidithiobacillus-like populations [61, 65, 68]. Indeed, previous reports showed that Thiobacillus species were widely found as the dominant member in some mining environments [1].

Reductive pathways of sulfate, which contained assimilatory sulfate reduction and dissimilatory sulfate reduction, ended with the formation of sulfide, and then entered into the biosynthesis of vital compounds. The coding genes for key enzymes of these processes were most closely related to Acidithiobacillus-like and Thiobacillus-like populations.

\section{Tolerant mechanisms to the extremely acidic environments}

We also focused on the research on extremely acidic environments, mainly because of the particularity of survival conditions that are harmful to most organisms and the adaptability of acidophiles that survive in these environments. Leach solution with chemical components which favor the formation of extremely acidic environments was sprayed onto the tailing dump, thus microbial species in the mine tailings probably possess the environmental adaptive capabilities.

\section{Heavy metal resistance}

Functional abundance profile analysis based on COG categories showed that COG categories [L] (Replication, recombination and repair; 9.44\%) were overrepresented in the metagenome dataset (Fig. 1). A reasonable explanation was that the concentration of toxic substance (e.g., heavy metals) in the extremely environment was higher than that of other 'normal' environments (e.g., agricultural soil), resulting in the accelerating rate of DNA injury $[2,69]$.

The bioleaching system is rich in high concentration of toxic metal elements such as copper, mercury, zinc, arsenic, cadmium and cobalt $[33,37]$. Microbes in this environment might possess certain resistance systems to responses to the heavy concentrations of metal ions. Energy-dependent efflux pumps, to a large extent, play a crucial role in toxic metal ion resistance, including ATPases and other chemiosmotic ion/proton exchangers; on the other hand, fewer mechanisms associated with enzymatic transformation such as oxidation, reduction, methylation and demethylation, which converts metal ions from more toxic to less toxic forms, and metal-binding proteins (e.g., metallothionein, protein chaperone and periplasmic binding protein) are also important for microbes to adapt the environmental conditions [70]. Researches showed that the microbial community in copper mine tailings harbored a variety of heavy metal resistance systems, such as ars operon arsenate resistance/regulation (arsC, COG1393; arsR, COG0640; arsB, COG1055; arsH, COG0431), mer operon mercuric resistance/regulation (merR, COG0789; merA, COG1249; merP, NOG79562; merT, NOG11562; merC, NOG56224; merD, NOG40540), CzcD-like cobalt-zinc-cadmium efflux $(c z c D, C O G 1230)$ and CzcABC cobalt-zinc-cadmium efflux ( $c z c A$, COG3696; $c z c B$, NOG01644; $c z c C$, NOG1 9426) (Additional file 6). And the majority of sequences associated with heavy metal resistance were assigned to Acidithiobacillus-like and Thiomonas-like microorganisms. Our results probably supported the viewpoint that microbial community in bioleaching heap adopted aforementioned mechanisms to cope with heavy metal stress.

\section{Low $\mathrm{pH}$ adaptation}

Given that microorganisms inhabit the acidic environments, the resident microbes may possess several strategies to maintain the circumneutral intracellular $\mathrm{pH}$. In order to acclimatize themselves to the extreme acidic environments, acidophiles share various structural and functional characteristics [17], mainly including (i) reversed membrane potential $(\Delta \Psi)$ generated by a Donnan potential that creates a chemiosmotic barrier to inhabit the influx of protons; (ii) highly impermeable membranes that restrict proton influx into the cytoplasm; and (iii) active secondary transporters that drives transport by utilizing the transmembrane electrochemical gradient of protons or sodium ions. Functional abundance profile analysis dependent on COG catalogues indicated that the potassium-efflux system proteins including potassiumtransporting ATPase subunit A (KdpA; COG2060), subunit B (KdpB; COG2216) and subunit C (KdpC; COG2 156), voltage-gated potassium channels proteins (COG12 26 and COG0667) and proton antiporters (CPA) (COG3 263/COG0025, COG0475/COG1226 and COG3004) were found in the metagenome. Most microorganisms having those genes shared sequence identity with the Acidithiobacillus-related sequences (Additional file 6). A large amount of associated genes showed that one potential mechanism to generate a reversed $\Delta \Psi$ was performed by potassiumtransporting ATPases [17]. In addition, several genes encoding plasma-membrane proton-efflux P-type ATPase (COG0474), which were presumed to exclude intracellular redundant protons, were detected in the metagenome.

\section{Organic solvent tolerance}

Organic solvents contain a large number of compounds with different kinds of chemical structures, e.g., benzene rings as well as aliphatic alcohols. Many of these compounds are greatly harmful to all life forms including humans, animals, plants and microorganisms [71]. They accumulate in cell membranes and undermine membrane integrity, resulting in the functional loss of membrane as the permeability barrier and energy transducer, and further leading to the alteration of intracellular $\mathrm{pH}$ and membrane electrical potential, cellar metabolism disorder, 
growth inhibition and even, eventually cell death [72-74]. Both in Gram-negative and Gram-positive microorganisms, there are common resistant mechanisms including energydependent active efflux pumps, cis-to-trans isomerization of unsaturated fatty acids mediated by cis-trans isomerase and changes in phospholipid head groups, generation of membrane vesicles transferring toxic compounds, and the change rate of phospholipid biosynthesis to expedite modify process [75].

Given that extractant was used in metal extraction industry [76], microorganisms exposed to Lix984n (an organic extractant), which was a potential substrate for RND efflux pumps [77], might harbor the stress-response strategies to cope with organic solvents in the bioleaching system. As a member of RND family proteins, the organic solvent efflux pump composed of AcrB (transporter AcrB/ AcrD/AcrF family protein; COG0841), AcrA (RND family efflux transporter MFP subunit; COG0845), and TolC (outer membrane efflux protein; COG1538) was presumed to transfer Lix984n as a potential substrate. Most of those CDSs were identified as Acidithiobacillus-like and Thiomonas-like sequences. Furthermore, a variety of COGs associated with toluene resistance, i.e., $\mathrm{ABC}$ transport system, including toluene tolerance protein (COG2854), Mce-related protein (substrate-binding protein) (NOG02063), toluene tolerance protein $\mathrm{Ttg} 2 \mathrm{~B}$ (permease protein) (COG0767) and toluene tolerance protein Ttg2A (ATPbinding protein) (COG1127), were identified in the metagenome. Moreover, functional abundance profile analysis based on COGs revealed that COG2067 (aromatic hydrocarbon degradation membrane protein) and COG1452 (organic solvent tolerance protein) were identified.

\section{Detoxification of hydroxyl radicals}

The reaction of ferrous iron (Fe(II)) with oxygen (Fenton reaction) could lead to the generation of hydroxyl radicals, which might cause the damage of biological macromolecules $[11,12]$. One strategy of avoiding the production of damaging free radicals is that electrons from Fe(II) substrates could be removed primarily using an outer membrane cytochrome $c$ [11]. In addition, evidence showed that the enzymatic detoxification of hydroxyl radicals was identified in Leishmania chagasi and L. donovani [78]. Based on sequence identities (30\% identity cut-off; Evalue $\leq 1 \mathrm{e}^{-5}$ ), sequence homologs for those peroxiredoxin coding genes (LcPxn1, LcPxn3 and LdPxn1) were mostly assigned to a Leptospirillum-like populations, indicating its key role in the detoxification of hydroxyl radicals. Moreover, other genes encoding antioxidant proteins such as peroxidase/catalase (COG0376) were also identified in the metagenome. Herein, the majority of sequences were assigned to the Euryarchaeota order Thermoplasmataleslike populations, especially Thermoplasmatales archaeon I-plasma-like and Thermoplasmatales archaeon Gpl-like groups, supporting the previous results that some extremely acidophilic genera belonging to order Thermoplasmatales were regularly found in bioleaching environments $[79,80]$.

\section{Conclusions}

The properties of environments, especially extremely acidic, oligotrophic and heavy metals containing bioleaching heap discussed in this study, shape the microbial community composition and function. Metabolic activities occur in the microbial community, conferring the role as a recycler of substance circulation in the bioleaching system and even in nature. Besides, whether environmental microorganisms harbor a suit of genes involving the response mechanisms is probably as a determinant factor to adapt the particular environmental conditions. Microorganisms in the extremely acidic environments have to cope with environmental stresses to survive and proliferate, before they can perform their functions in the bioleaching system.

\section{Methods}

Sampling, DNA extraction and metagenome sequencing The sampling site is located in Dexing Copper Mine, China. We obtained the permission from Jiangxi Copper Company Limited to access the site and to sample there. Metal recovery from low-grade copper tailings is performed via spraying the bioleaching solution onto the tailings dump. A detailed description of the sampling site and tailings, including $\mathrm{pH}$, redox potential, sulfur compounds, ferrous iron, and metal ions, as well as a preliminary study of the microbial community diversity of the site have been reported [37].

In order to further explore the microbial community composition and function of mine tailings during the bioleaching process, samples were collected from surface-layer (approximately $20 \mathrm{~cm}$ depth) of bioleaching heap. Before DNA extraction, samples were washed with distilled water ( $\mathrm{pH} 2.0 ; 3-4$ times), and then filtered through a $0.22-\mu \mathrm{m}$ pore-size filter membrane using the vacuum filtration device. Subsequently, the metagenomic DNA was extracted according to the previous method $[81,82]$. The purified DNA sample was then used to construct the shotgun library ( $300 \mathrm{bp}$ average insert size), which was sequenced (250 bp paired-end reads) using an Illumina MiSeq sequencer located at our own laboratory [83].

\section{Bioinformatics analysis for metagenome data}

In order to filter the data for high-quality (HQ), the quality control (QC) of sequencing reads was performed using NGS QC Toolkit v2.3.1 (cutoff read length for HQ, $70 \%$; cutoff quality score, 20) [84].

For contig level analysis, the HQ sequencing data were assembled using MetaVelvet 1.2.02 and SOAPdenovo 
with various k-mer (ranging from 31 to 121; the stepsize is 10). Subsequently, diverse contig sequences were then clustered using the program CD-HIT-EST with a sequence identity threshold of $97 \%$ [85], and assemblies $(\geq 300 \mathrm{bp}$ ) were retained for further analyses. Considering the fact that bacteria were predominant in this environment, gene prediction was performed using MetaGeneAnnotator, which is a gene-finding program for prokaryote and phage $[86,87]$. To acquire the taxonomic classification of the protein coding gene sequences $[2,49,88]$, the NCBI non-redundant $(\mathrm{nr})$ comparison results were parsed using MEGAN with the lowest ancestor algorithm [89, 90]. Besides, the putative protein-coding sequences were compared ( $e$-value threshold, $10^{-5}$ ) against the databases including NCBI-nr, the extended COG (Clusters of Orthologous Groups) [28] and KEGG (Kyoto Encyclopedia of Genes and Genomes) to obtain their functional annotation. The relative abundance of a given taxon, as well as gene sequence against the particular databases (i.e., NR, COG and KEGG) was calculated according to the previous analytical method in other metagenomic studies [49].

\section{Availability of supporting data}

The datasets supporting the results of this article are available in the MG-RAST (the Metagenomics RAST) repository. The assembled metagenome dataset from an Illumina MiSeq was deposited at MG-RAST under the accession number of 4664533.3 (http://metagenomics.anl.gov/linkin.cgi?metagenome=4664533.3). Additionally, other supporting datasets are available within the additional files of this article.

\section{Additional files}

\section{Additional file 1: Summary of metagenome dataset of samples} from the surface-layer mine tailings. (DOC $40 \mathrm{~kb}$ )

Additional file 2: Summary of major phylotypes of microorganism communities in copper mine tailings using the program MEGAN. (XLSX $13 \mathrm{~kb})$

Additional file 3: The viral composition in the metagenome collected from bioleaching heap. (TIF $423 \mathrm{~kb}$ )

Additional file 4: Phylogenetic tree at the phylum level on the basis of metagenome dataset. The data was compared to M5NR using a maximum e-value of $1 \mathrm{e}^{-5}$, a minimum identity of $60 \%$, and a minimum alignment length of 15 measured in aa for protein and bp for RNA databases. In addition, display leaf weights as stacked bar, maximum level is class, color by phylum. (TIF $3380 \mathrm{~kb}$ )

Additional file 5: Detailed information of CDSs associated with microbial carbon fixation, nitrogen metabolism, sulfur metabolism and ferrous iron oxidation in metagenome dataset. (XLSX $26 \mathrm{~kb}$ )

Additional file 6: Key genes encoding enzymes related to heavy metal resistance and low pH tolerance. (DOCX $31 \mathrm{~kb}$ )

\section{Authors' contributions}

$X Z, Y L, X L$ and $H Y$ conceived and designed the experiments. $X Z$ and JN performed the experiments. $X Z$ analyzed the data. $X Z$ wrote the manuscript. $X \mathrm{~L}$ and $\mathrm{HY}$ revised the manuscript. All authors read and approved the final version of the manuscript.

\section{Acknowledgements}

This work was supported by the National Natural Science Foundation of China (No. 31570113 and No. 41573072). In addition, we would like to thank Dr. Zhili He in University of Oklahoma for proofreading the manuscript.

Received: 7 August 2015 Accepted: 13 January 2016

Published online: 19 January 2016

\section{References}

1. Schippers A, Breuker A, Blazejak A, Bosecker K, Kock D, Wright TL. The biogeochemistry and microbiology of sulfidic mine waste and bioleaching dumps and heaps, and novel Fe (II)-oxidizing bacteria. Hydrometallurgy. 2010;104(3-4):342-50.

2. Chen LX, Li JT, Chen YT, Huang LN, Hua ZS, Hu M, et al. Shifts in microbial community composition and function in the acidification of a lead/zinc mine tailings. Environ Microbiol. 2013;15(9):2431-44.

3. Bosecker K, Mengel-Jung G, Schippers A. Geomicrobiological risk assessment of abandoned mining sites. In: Tsezos M, Hatzikioseyian A, Remoundaki E, editors. Biohydrometallurgy: A Sustainable Technology in Evolution, Part 1. Athens: University of Athens; 2004. p. 585-93.

4. Breuker A, Blazejak A, Bosecker K, Schippers A. Diversity of iron oxidizing bacteria from various sulfidic mine waste dumps. Adv Mater Res. 2009;71-73:47-50.

5. Kock D, Schippers A. Quantitative microbial community analysis of three different sulfidic mine tailing dumps generating acid mine drainage. Appl Environ Microbiol. 2008;74(16):5211-9.

6. Kock D, Schippers A. Geomicrobiological investigation of two different mine waste tailings generating acid mine drainage. Hydrometallurgy. 2006:83(1-4):167-75.

7. Liljeqvist M, Ossandon FJ, González C, Rajan S, Stell A, Valdes J, et al. Metagenomic analysis reveals adaptations to a cold-adapted lifestyle in a low-temperature acid mine drainage stream. FEMS Microbiol Ecol. 2015;91(4):fiv011.

8. Huang LN, Zhou WH, Hallberg KB, Wan C, Li J, Shu WS. Spatial and temporal analysis of the microbial community in the tailings of a $\mathrm{Pb}-\mathrm{Zn}$ mine generating acidic drainage. Appl Environ Microbiol. 2011;77(15):5540-4.

9. Liu J, Hua Z, Chen L, Kuang J, Li S, Shu W, et al. Correlating microbial diversity patterns with geochemistry in an extreme and heterogeneous environment of mine tailings. Appl Environ Microbiol. 2014;80(12):3677-86.

10. Demergasso CS, Galleguillos PAP, Escudero LVG, Zepeda VJA, Castillo D, Casamayor EO. Molecular characterization of microbial populations in a lowgrade copper ore bioleaching test heap. Hydrometallurgy. 2005;80(4):241-53.

11. Bonnefoy V, Holmes DS. Genomic insights into microbial iron oxidation and iron uptake strategies in extremely acidic environments. Environ Microbiol. 2012;14(7):1597-611

12. Touati D. Iron and oxidative stress in bacteria. Arch Biochem Biophys. 2000; 373(1):1-6.

13. López-Archilla Al, Marin I, Amils R. Microbial community composition and ecology of an acidic aquatic environment: the Tinto River, Spain. Microb Ecol. 2001:41(1):20-35.

14. González-Toril E, Llobet-Brossa E, Casamayor EO, Amann R, Amils R. Microbial ecology of an extreme acidic environment, the Tinto River. Appl Environ Microbiol. 2003;69(8):4853-65.

15. Sánchez-Andrea I, Rodríguez N, Amils R, Sanz JL. Microbial diversity in anaerobic sediments at Rio Tinto, a naturally acidic environment with a high heavy metal content. Appl Environ Microbiol. 2011;77(17):6085-93.

16. Franke S, Rensing C. Acidophiles: mechanisms to tolerate metal and acid toxicity. In: Gerday C, Glansdorff N, editors. Physiology and biochemistry of extremophiles. Washington, DC: ASM press; 2007. p. 271-7.

17. Baker-Austin C, Dopson M. Life in acid: pH homeostasis in acidophiles. Trends Microbiol. 2007;15(4):165-71.

18. Tyson GW, Chapman J, Hugenholtz P, Allen EE, Ram RJ, Richardson PM, et al. Community structure and metabolism through reconstruction of microbial genomes from the environment. Nature. 2004;428(6978):37-43. 
19. Sabater S, Buchaca T, Cambra J, Catalan J, Guasch H, Ivorra N, et al. Structure and function of benthic algal communities in an extremely acid river. J Phycol. 2003;39(3):481-9.

20. Brierley CL. Bacterial succession in bioheap leaching. Hydrometallurgy. 2001; 59(2-3):249-55.

21. Johnson SS, Chevrette MG, EhImann BL, Benison KC. Insights from the metagenome of an acid salt lake: the role of biology in an extreme depositional environment. PLoS One. 2015;10(4):e122869.

22. Handelsman J. Metagenomics: application of genomics to uncultured microorganisms. Microbiol Mol Biol Rev. 2004;68(4):669-85.

23. Friedrich MW. Stable-isotope probing of DNA: insights into the function of uncultivated microorganisms from isotopically labeled metagenomes. Curr Opin Biotechnol. 2005;17(1):59-66.

24. Riesenfeld CS, Schloss PD, Handelsman J. Metagenomics: genomic analysis of microbial communities. Annu Rev Genet. 2004;38(1):525-52.

25. Streit WR, Schmitz RA. Metagenomics - the key to the uncultured microbes. Curr Opin Microbiol. 2004;7(5):492-8.

26. Hua ZS, Han YJ, Chen LX, Liu J, Hu M, Li SJ, et al. Ecological roles of dominant and rare prokaryotes in acid mine drainage revealed by metagenomics and metatranscriptomics. ISME J. 2015;9(6):1280-94.

27. Schloss PD, Handelsman J. Metagenomics for studying unculturable microorganisms: cutting the Gordian knot. Genome Biol. 2005;6(8):229.

28. Franceschini A, Szklarczyk D, Frankild S, Kuhn M, Simonovic M, Roth A, et al. STRING v9. 1: protein-protein interaction networks, with increased coverage and integration. Nucleic Acids Res. 2013;41(D1):D808-15.

29. Baker BJ, Banfield JF. Microbial communities in acid mine drainage. FEMS Microbiol Ecol. 2003;44(2):139-52.

30. Johnson DB, Hallberg KB. The microbiology of acidic mine waters. Res Microbiol. 2003;154(7):466-73.

31. Hallberg KB. New perspectives in acid mine drainage microbiology. Hydrometallurgy. 2010;104(3-4):448-53.

32. Hallberg KB, González-Toril E, Johnson DB. Acidithiobacillus ferrivorans, sp nov.; facultatively anaerobic, psychrotolerant iron-, and sulfur-oxidizing acidophiles isolated from metal mine-impacted environments. Extremophiles. 2010;14(1):9-19.

33. Valdés J, Pedroso I, Quatrini R, Dodson RJ, Tettelin H, Blake R, et al. Acidithiobacillus ferrooxidans metabolism: from genome sequence to industrial applications. BMC Genomics. 2008;9(1):597.

34. Fancello L, Trape S, Robert C, Boyer M, Popgeorgiev N, Raoult D, et al. Viruses in the desert: a metagenomic survey of viral communities in four perennial ponds of the Mauritanian Sahara. ISME J. 2013;7(2):359-69.

35. Adriaenssens EM, Van Zyl L, De Maayer P, Rubagotti E, Rybicki E, Tuffin M, et al. Metagenomic analysis of the viral community in Namib Desert hypoliths. Environ Microbiol. 2015;17(2):480-95.

36. Breitbart M, Salamon P, Andresen B, Mahaffy JM, Segall AM, Mead D, et al. Genomic analysis of uncultured marine viral communities. Proc Natl Acad Sci USA. 2002;99(22):14250-5.

37. Yin H, Qiu G, Wu L, Xie M, Zhou J, Dai Z, et al. Microbial community diversity and changes associated with a mine drainage gradient at the Dexing copper mine, China. Aquat Microb Ecol. 2008;51(1):67-76.

38. Williams KP, Kelly DP. Proposal for a new class within the phylum Proteobacteria, Acidithiobacillia classis nov., with the type order Acidithiobacillales, and emended description of the class Gammaproteobacteria. Int J Syst Evol Microbiol. 2013;63(8):2901-6.

39. Berg IA, Kockelkorn D, Ramos-Vera WH, Say RF, Zarzycki J, Hügler M, et al. Autotrophic carbon fixation in archaea. Nat Rev Microbiol. 2010;8(6):447-60.

40. Levicán G, Ugalde JA, Ehrenfeld N, Maass A, Parada P. Comparative genomic analysis of carbon and nitrogen assimilation mechanisms in three indigenous bioleaching bacteria: predictions and validations. BMC Genomics. 2008;9(1):581.

41. Shively JM, van Keulen G, Meijer WG. Something from almost nothing: carbon dioxide fixation in chemoautotrophs. Annu Rev Microbiol. 1998;52:191-230.

42. Guo X, Yin H, Cong J, Dai Z, Liang Y, Liu X. RubisCO gene clusters found in a metagenome microarray from acid mine drainage. Appl Environ Microbiol. 2013;79(6):2019-26.

43. Bertin PN, Heinrich-Salmeron A, Pelletier E, Goulhen-Chollet F, ArsènePloetze F, Gallien S, et al. Metabolic diversity among main microorganisms inside an arsenic-rich ecosystem revealed by meta-and proteo-genomics. ISME J. 2011;5(11):1735-47.

44. Hügler M, Wirsen CO, Fuchs G, Taylor CD, Sievert SM. Evidence for autotrophic $\mathrm{CO}_{2}$ fixation via the reductive tricarboxylic acid cycle by members of the $\varepsilon$ subdivision of proteobacteria. J Bacteriol. 2005; 187(9):3020-7.

45. Berg IA. Ecological aspects of the distribution of different autotrophic $\mathrm{CO}_{2}$ fixation pathways. Appl Environ Microbiol. 2011;77(6):1925-36.

46. Aoshima M, Ishii M, Igarashi Y. A novel enzyme, citryl-CoA synthetase, catalysing the first step of the citrate cleavage reaction in Hydrogenobacter thermophilus TK-6. Mol Microbiol. 2004;52(3):751-61.

47. Aoshima M, Ishii M, Igarashi Y. A novel enzyme, citryl-CoA lyase, catalysing the second step of the citrate cleavage reaction in Hydrogenobacter thermophilus TK-6. Mol Microbiol. 2004;52(3):763-70.

48. Hügler M, Huber $\mathrm{H}$, Molyneaux SJ, Vetriani $\mathrm{C}$, Sievert SM. Autotrophic $\mathrm{CO}_{2}$ fixation via the reductive tricarboxylic acid cycle in different lineages within the phylum Aquificae: evidence for two ways of citrate cleavage. Environ Microbiol. 2007;9(1):81-92.

49. Chen LX, Hu M, Huang LN, Hua ZS, Kuang JL, Li SJ, Shu WS. Comparative metagenomic and metatranscriptomic analyses of microbial communities in acid mine drainage. ISME J. 2015:9(7):1579-1592

50. He Z, Xu M, Deng Y, Kang S, Kellogg L, Wu L, et al. Metagenomic analysis reveals a marked divergence in the structure of belowground microbial communities at elevated $\mathrm{CO}_{2}$. Ecol Lett. 2010;13(5):564-75.

51. Mason OU, Scott NM, Gonzalez A, Robbins-Pianka A, Bælum J, Kimbrel J, et al. Metagenomics reveals sediment microbial community response to Deepwater Horizon oil spill. ISME J. 2014;8(7):1464-75.

52. Méndez-García C, Peláez Al, Mesa V, Sánchez J, Golyshina OV, Ferrer M. Microbial diversity and metabolic networks in acid mine drainage habitats. Front Microbiol. 2015;6:475

53. Jones CM, Stres B, Rosenquist M, Hallin S. Phylogenetic analysis of nitrite, nitric oxide, and nitrous oxide respiratory enzymes reveal a complex evolutionary history for denitrification. Mol Biol Evol. 2008; 25(9):1955-66.

54. Weber KA, Achenbach LA, Coates JD. Microorganisms pumping iron: anaerobic microbial iron oxidation and reduction. Nat Rev Microbiol. 2006 4(10):752-64.

55. Emerson D, Fleming EJ, McBeth JM. Iron-oxidizing bacteria: an environmental and genomic perspective. Annu Rev Microbiol. 2010;64:561-83.

56. Hedrich S, Schlömann M, Johnson DB. The iron-oxidizing proteobacteria. Microbiology. 2011;157(6):1551-64.

57. Cárdenas JP, Ortiz R, Norris PR, Watkin E, Holmes DS. Reclassification of 'Thiobacillus prosperus' Huber and Stetter 1989 as Acidihalobacter prosperus gen. nov., sp. nov., a member of the family Ectothiorhodospiraceae. Int J Syst Evol Microbiol. 2015;65(10):3641-4.

58. Bird LJ, Bonnefoy $V$, Newman DK. Bioenergetic challenges of microbial iron metabolisms. Trends Microbiol. 2011;19(7):330-40.

59. Ilbert M, Bonnefoy V. Insight into the evolution of the iron oxidation pathways. Biochim Biophys Acta-Bioenerg. 2013;1827(2):161-75.

60. Castelle CJ, Roger M, Bauzan M, Brugna M, Lignon S, Nimtz M, et al. The aerobic respiratory chain of the acidophilic archaeon Ferroplasma acidiphilum: A membrane-bound complex oxidizing ferrous iron. Biochim Biophys Acta-Bioenerg. 2015;1847(8):717-28.

61. Yin H, Zhang X, Li X, He Z, Liang Y, Guo X, et al. Whole-genome sequencing reveals novel insights into sulfur oxidation in the extremophile Acidithiobacillus thiooxidans. BMC Microbiol. 2014;14:179.

62. Johnson DB, Hallberg KB. Carbon, iron and sulfur metabolism in acidophilic micro-organisms. Adv Microb Physiol. 2009;54:201-55.

63. Dopson M, Johnson DB. Biodiversity, metabolism and applications of acidophilic sulfur-metabolizing microorganisms. Environ Microbiol. 2012; 14(10):2620-31.

64. Schippers A, Sand W. Bacterial leaching of metal sulfides proceeds by two indirect mechanisms via thiosulfate or via polysulfides and sulfur. Appl Environ Microbiol. 1999:65(1):319-21.

65. Chen L, Ren Y, Lin J, Liu X, Pang X, Lin J. Acidithiobacillus caldus sulfur oxidation model based on transcriptome analysis between the wild type and sulfur oxygenase reductase defective mutant. PLoS One. 2012;7(9): e39470.

66. Urich T, Bandeiras TM, Leal SS, Rachel R, Albrecht T, Zimmermann P, et al. The sulphur oxygenase reductase from Acidianus ambivalens is a multimeric protein containing a low-potential mononuclear non-haem iron centre. Biochem J. 2004:381(1):137-46.

67. Li M, Chen Z, Zhang P, Pan X, Jiang C, An X, et al. Crystal structure studies on sulfur oxygenase reductase from Acidianus tengchongensis. Biochem Biophys Res Commun. 2008;369(3):919-23. 
68. Quatrini R, Appia-Ayme C, Denis Y, Jedlicki E, Holmes DS, Bonnefoy V. Extending the models for iron and sulfur oxidation in the extreme acidophile Acidithiobacillus ferrooxidans. BMC Genomics. 2009;10(1):394.

69. Silver S, Phung LT. Bacterial heavy metal resistance: new surprises. Annu Rev Microbiol. 1996;50:753-89.

70. Silver S, Phung LT. A bacterial view of the periodic table: genes and proteins for toxic inorganic ions. J Ind Microbiol Biotechnol. 2005;32(11-12): 587-605.

71. Segura A, Molina L, Fillet S, Krell T, Bernal P, Muñoz-Rojas J, et al. Solvent tolerance in Gram-negative bacteria. Curr Opin Biotechnol. 2012;23(3):415-21.

72. Sardessai YN, Bhosle S. Organic solvent-tolerant bacteria in mangrove ecosystem. Curr Sci. 2002;82(6):622-3.

73. Heipieper HJ, Neumann G, Cornelissen S, Meinhardt F. Solvent-tolerant bacteria for biotransformations in two-phase fermentation systems. Appl Microbiol Biotechnol. 2007:74(5):961-73.

74. Sikkema J, de Bont JA, Poolman B. Mechanisms of membrane toxicity of hydrocarbons. Microbiol Rev. 1995;59(2):201-22.

75. Torres S, Pandey A, Castro GR. Organic solvent adaptation of Gram positive bacteria: Applications and biotechnological potentials. Biotechnol Adv. 2011; 29(4):442-52.

76. Zhou Z, Fang Y, Li Q, Yin H, Qin W, Liang Y, et al. Global transcriptional analysis of stress-response strategies in Acidithiobacillus ferrooxidans ATCC 23270 exposed to organic extractant-Lix984n. World J Microbiol Biotechnol. 2012;28(3):1045-55.

77. Liu J, Qiu G, Ge Y, Xu J. Extraction of copper from bacterial leach solution using Lix984. Trans Nonferrous Met Soc China. 2002;12(2):313-6.

78. Barr SD, Gedamu L. Cloning and characterization of three differentially expressed peroxidoxin genes from Leishmania chagasi. Evidence for an enzymatic detoxification of hydroxyl radicals. J Biol Chem. 2001;276(36):34279-87.

79. Wheaton G, Counts J, Mukherjee A, Kruh J, Kelly R. The confluence of heavy metal biooxidation and heavy metal resistance: implications for bioleaching by extreme thermoacidophiles. Minerals. 2015;5(3):397-451.

80. Barahona S, Dorador C, Zhang R, Aguilar P, Sand W, Vera M, et al. Isolation and characterization of a novel Acidithiobacillus ferrivorans strain from the Chilean Altiplano: attachment and biofilm formation on pyrite at low temperature. Res Microbiol. 2014;165(9):782-93.

81. Zhou J, Bruns MA, Tiedje JM. DNA recovery from soils of diverse composition. Appl Environ Microbiol. 1996;62(2):316-22

82. Deng J, Gu Y, Zhang J, Xue K, Qin Y, Yuan M, et al. Shifts of tundra bacterial and archaeal communities along a permafrost thaw gradient in Alaska. Mol Ecol. 2015;24(1):222-34.

83. Yin $\mathrm{H}$, Niu J, Ren $Y$, Cong J, Zhang $X$, Fan F, et al. An integrated insight into the response of sedimentary microbial communities to heavy metal contamination. Sci Rep. 2015;5:14266

84. Patel RK, Jain M. NGS QC Toolkit: a toolkit for quality control of next generation sequencing data. PLoS One. 2012;7(2):e30619.

85. Li W, Godzik A. Cd-hit: a fast program for clustering and comparing large sets of protein or nucleotide sequences. BIOINFORMATICS. 2006;22(13): 1658-9.

86. Noguchi H, Park J, Takagi T. MetaGene: prokaryotic gene finding from environmental genome shotgun sequences. Nucleic Acids Res. 2006;34(19): 5623-30.

87. Noguchi H, Taniguchi T, Itoh T. MetaGeneAnnotator: detecting speciesspecific patterns of ribosomal binding site for precise gene prediction in anonymous prokaryotic and phage genomes. DNA Res. 2008;15(6):387-96.

88. Urich T, Lanzén A, Qi J, Huson DH, Schleper C, Schuster SC. Simultaneous assessment of soil microbial community structure and function through analysis of the meta-transcriptome. PLoS One. 2008;3(6):e2527.

89. Huson DH, Auch AF, Qi J, Schuster SC. MEGAN analysis of metagenomic data. Genome Res. 2007:17(3):377-86.

90. Huson DH, Mitra S, Ruscheweyh H, Weber N, Schuster SC. Integrative analysis of environmental sequences using MEGAN4. Genome Res. 2011; 21(9):1552-60.

\section{Submit your next manuscript to BioMed Central and we will help you at every step:}

- We accept pre-submission inquiries

- Our selector tool helps you to find the most relevant journal

- We provide round the clock customer support

- Convenient online submission

- Thorough peer review

- Inclusion in PubMed and all major indexing services

- Maximum visibility for your research

Submit your manuscript at www.biomedcentral.com/submit

) Biomed Central 